Bolm. 200Z., Unio. S. Parlo

$7: 213-823,1988$

\title{
RELAÇÃO DO COMPRIMENTO DOS DIAS COM A ATIVIDADE REPRODUTIVA EM SICALIS FLAVEOLA (LINNAEUS, 1766) (PASSERIFORMES, EMBERIZIDAE).
}

LUIZ OCTAVIO MARCONDES MACHADO

Instituto de Biologia, Departanento de Zoolo gia da Universidade Estadual de Campinas, Ca⿳⺈ pinas, São Paulo. (recebido em 12/XI/1982)

RESUMO - Procurou-se estabelecer uma relação entre $\rho$ comprimento dos dias durante 0 ano e o comportamento reprodutivo em Sicalis flaveola (Linnaeus, 1766). Foran observados ca sais em cativeiro na Fundação Parque Zoológico de São Paulo e na natureza na Faz. Jatibaia, Campinas, SP. Pode-se verifi car a ocorrência de inatividade reprodutiva, nos casais em cativeiro, a partir de dias de comprimentos de 11:05 h e um reinício a partir de dias com mais de $10: 55 \mathrm{~h}$. Na natureza a inatividade teve início em dias com menos de 11:55 h e reiní cio em dias com mais de 11:00 h. Pelos dados apresentados, le vando-se em conta que a alimentação foi sempre a mesma parā os casais mantidos em cativeiro durante 0 ano todo, a variação do comprimento dos dias influiu na atividade reprodutiva desta espécie.

ABSTRACT - A relationship between the length of the day during the year and the reproductive behaviour of sicalis flaveola (Linnaeus, 1766) was established. Some couples were observed in captivity at the Fundação Parque Zoológico de São Paulo and others at the Jatibaia Farm, Campinas, Sào Pau 10. The occurrence of reproductive inactivity was verified in the couples in captivity, in days of 11:05 hours lenght and reinitiating in days with more than 10:55 hours. In natu re the inactivity began in days with less than 11:55 hours, reinitiating in days with more than 11:00 hours. By the presented figures, and taking into consideration that the ali mentation supplied was the same for all couples kept in captivity during all the year, the variation of the length of the days influenced the reproductive activity of this spe cies. 


\section{INTRODUÇĀO}

o nümero de ninhadas produzidas por diferentes espé cies de pássaros em um ano é muito mais uma característica da área geográfica, do que de uma espécie em particular. Hou ve, portanto, uma influência dos estímulos ambientais, entrē eles o comprimento dos dias, com o desenvolvimento da atividade glandular. Esta influência capacitou as aves, de uma ma neira geral, a terem seus filhotes numa época do ano em que as condiçōes são mais favoráveis ao seu desenvolvimento (Wel ty, 1962; Lack, 1968).

Nas aves que habitam médias e altas latitudes, 0 fotoperiodo é o estimulador de maior importância no controle ime diato dos ciclos anuais. Os machos são mais sujeitos à fotoestimulação, pois esta induz a complementação da espermatogê nese (Immelmann, 1971).

Nas regiões tropicais, onde a variação do comprimento dos dias é relativamente menor que nas regiōes temperadas, a importância do fotoperíodo é questionada, embora em condi ções experimentais algumas espécies sofram influências da fô toestimulação (Immelmann, 1971).

Para se avaliar a influência desse fator ambiental em pássaros tropicais e subtropicais foi aqui estudada a atividade reprodutiva de Sicalis flaveola (Linnaeus, 1766), popularmente conhecido como canário-da-terra, em relação ao comprimento dos dias, em condições naturais.

\section{MATERIAIS E METODOS}

As observaçōes foram feitas em casais reproduzindo-se em cativeiro e na natureza. Em cativeiro as observações fo ram realizadas na Fundação Parque Zoológico de São Paulo com 16 casais de $S$. flaveola brasiliensis, mantidos em gaiolas

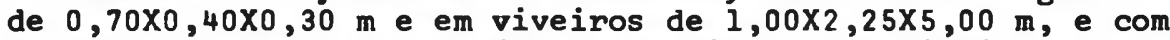
um casal de $S$. f. pelzelni que procriou em um viveiro de $1,00 \times 2,25 \times 2,50 \mathrm{~m}$. Na natureza, os estudos foram desenvolvi dos com 8 casais de $s$. $f$. brasiliensis renaturalizados, na Fazenda Jatibaia, município de Campinas, SP.

Os dados sobre os pássaros em cativeiro foram obtidos no período de julho de 1972 a abril de 1975 . Na natureza, as observaçōes foram efetuadas de julho de 1976 a março de 1978. Para os casais mantidos em cativeiro foram realizadas medidas do período de incubação de 25 oviposturas e de perma nência dos filhotes no ninho de 15 ninhadas. Nos casais ob = servados na natureza foram feitas medidas do período de incu bação de 8 oviposturas e período de permanência dos filhotes no ninho de 9 ninhadas

As fases do comportamento reprodutivo foram relacionadas com a variação dos comprimentos dos dias, sendo estes dados fornecidos pelo Instituto Astronômico e Geofísico da USP .

A alimentação em cativeiro constou de alpiste (Phalaris canariensis), painço (Setaria italica), de uma mistura de pão seco moído, leite, almeirão (Chicorium intybus) pica- 
do, "Vitagold" (laboratōrio Tortuga), "Gebral" (Laboratório Lederle) e gema de ovo. Na época de cuidados con os filhotes foram fornecidas larvas de Tonebrio molitor e principalmente na época de ovipostura, cascas de ovo de galinha.

\section{RESULTADOS}

\section{Em cativeiro}

Foram considerados os dados de $s$. f. brasiliensis e $s$. f. pelzelni em conjunto. Observando-se os dados contidos nas figs. I a 4 pode-se verificar que há una inatividade reprodu tiva nos dias com menos de 11:05 horas e um reinício de ati= vidade a partir de comprimento de dias superiores a 10:55 ho ras. Com o aumento do comprimento dos dias, os casais estudā dos iniciaram a construção do ninho, podendo em seguida oco $\bar{r}$ rer a ovipostura e demais atividades do ciclo reprodutivo.

Um dos casais efetuou a ovipostura entre dias com comprimento de 10:45 horas a 10:43 horas, mas não houve nasci mento de filhotes.

Em alguns casais a construção do ninho se prolongou por grande parte do período reprodutivo, com altemancia de curtos períodos dedicados a carregar material para o ninho

\section{Na natureza}

Foram observados apenas casais de $s$. $f$. brasilieneis Em 5 dos casais estudados o período reprodutivo se estendeu por dias de comprimento de no maximo 11:55 horas. Levando-se em conta 2 casais, as atividades reiniciaram em dias de comprimento mínimo de 11:00 horas. Um dos casais observados obteve filhotes a partir de duas oviposturas iniciadas em dias com comprimento de $11: 34$ horas e 10:42 horas respectivamente. Os filhotes deixaram o ninho em dias de comprimento igual a 10.52 horas na primeira e de 10:53 horas na segunda (Fig. 5 a 7).

\section{DISCUSSĀO E CONCLUSÃO}

Em S. f. brasilieneic e S. f. pelselni, espécies tropi cais e subtropicais, parece existir uma nítida influência dō comprimento dos dias em relação ao ciclo reprodutivo.

Algumas espécies que habitam a América Central aparentemente sofrem pouca influéncia do fotoperíodo e procriam quando ocorre fartura de alimento (Skutch, 1950). Para s. flaveola em cativeiro e alimentação fornecida era sempre a mesma e ocorreram períodos de inatividade reprodutiva.

Aves cativas, de una maneira geral, têm un alto número de ninhadas sucessivas (Immelman, 1971); s. f. brasilieneio procria mais de uma vez por ano, mesmo em condições naturais. Segundo Lack (1968), este fato está mais relacionado às condiçoes propícias do ambiente, dependentes do comprimento dos dias, do que das características da própria espécie. 


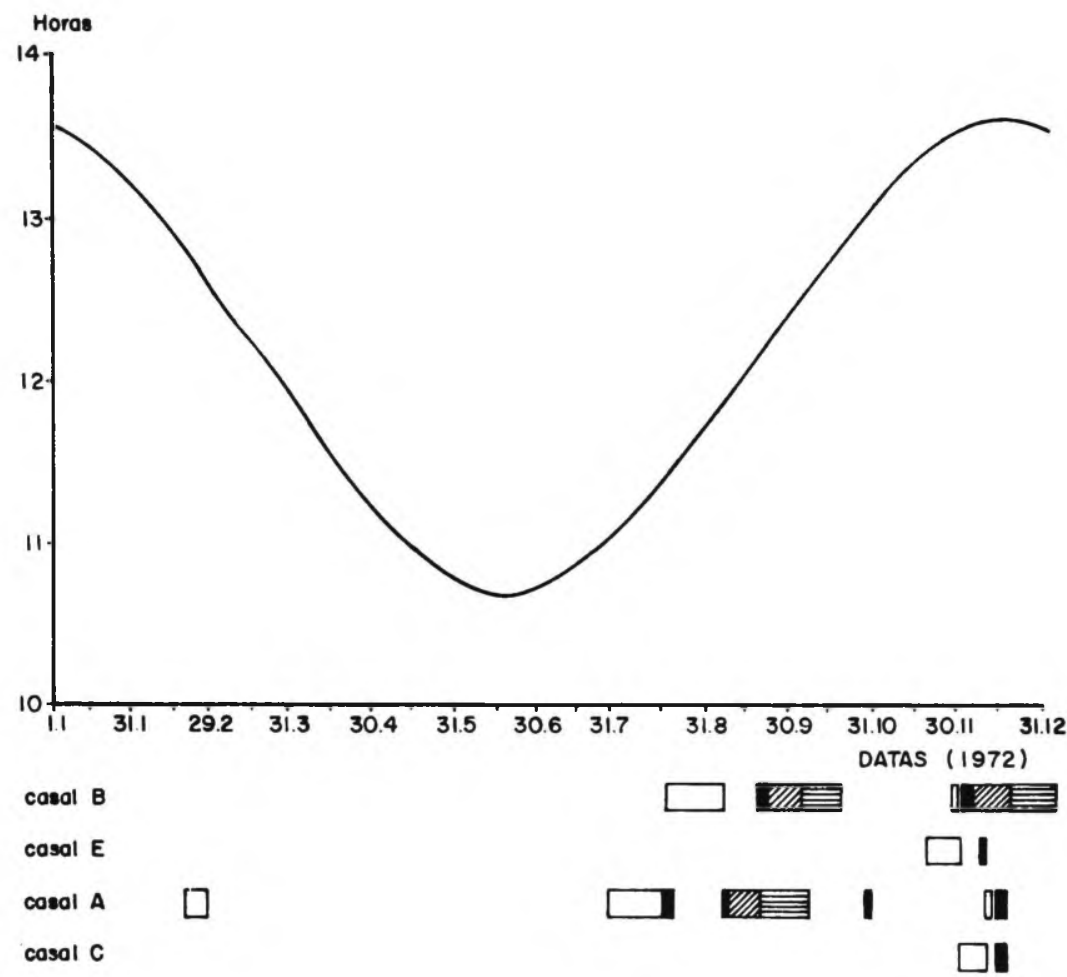

$\square$ construção do ninho

ovipostura

W incubaçōo

目 permanância dos filhotes no ninho

construçäo de ninho com frequiência baixa, näo fendo como sequiência a postura

Fig. 1 - Comportamento reprodutivo em cativeiro de casais de Sicalis flaveola brasiliensis, com exceção do casal $C$, que é de S. f. pelzelni, em funçäo do comprimento dos dias em 1972 


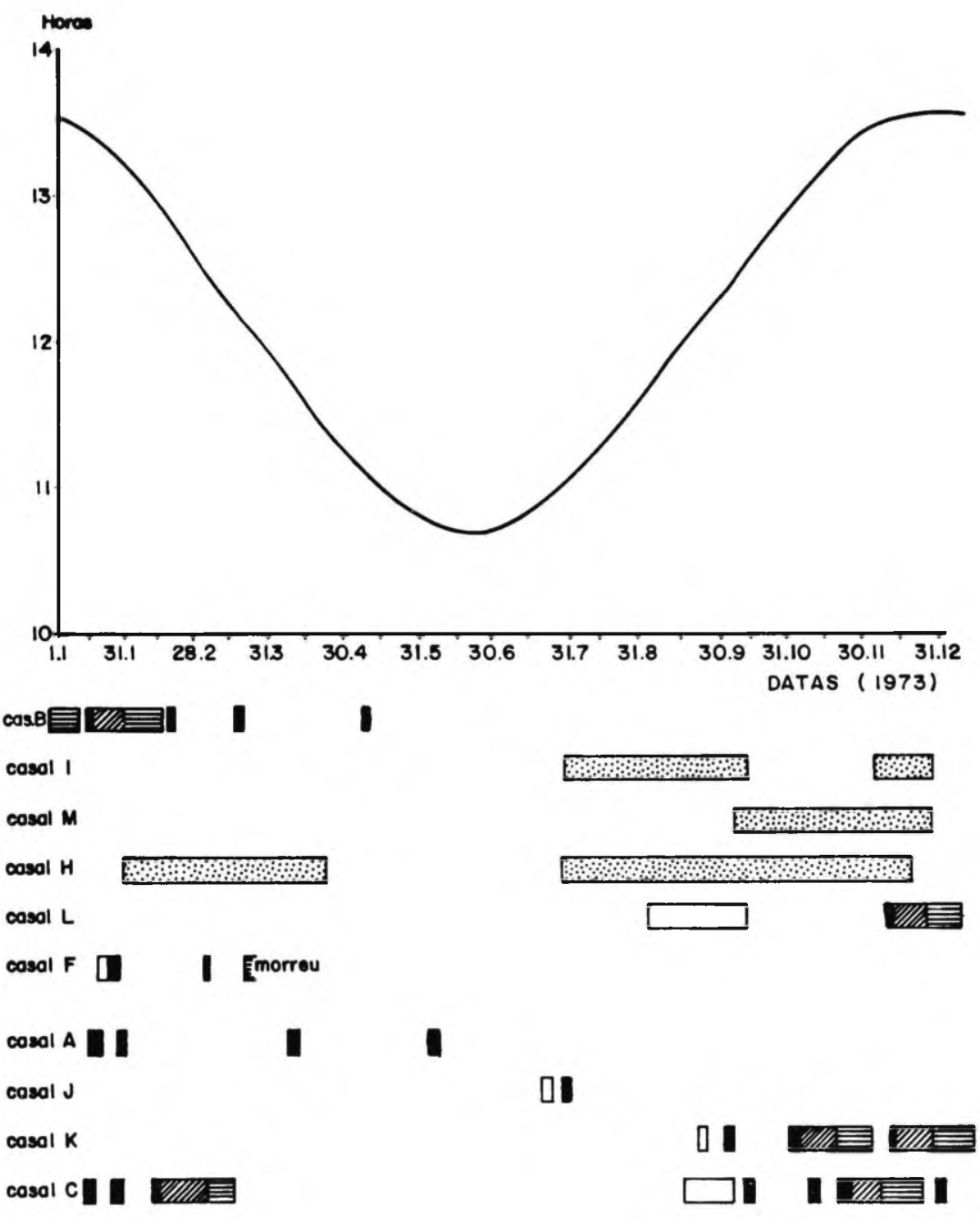

Fig. 2 - Comportamento reprodutivo em cativeiro de casais de s. f. brasiliensis, com exceção do casal $C$, que é de $S$. f. pelselni, em função do comprimento dos dias em 1973. 


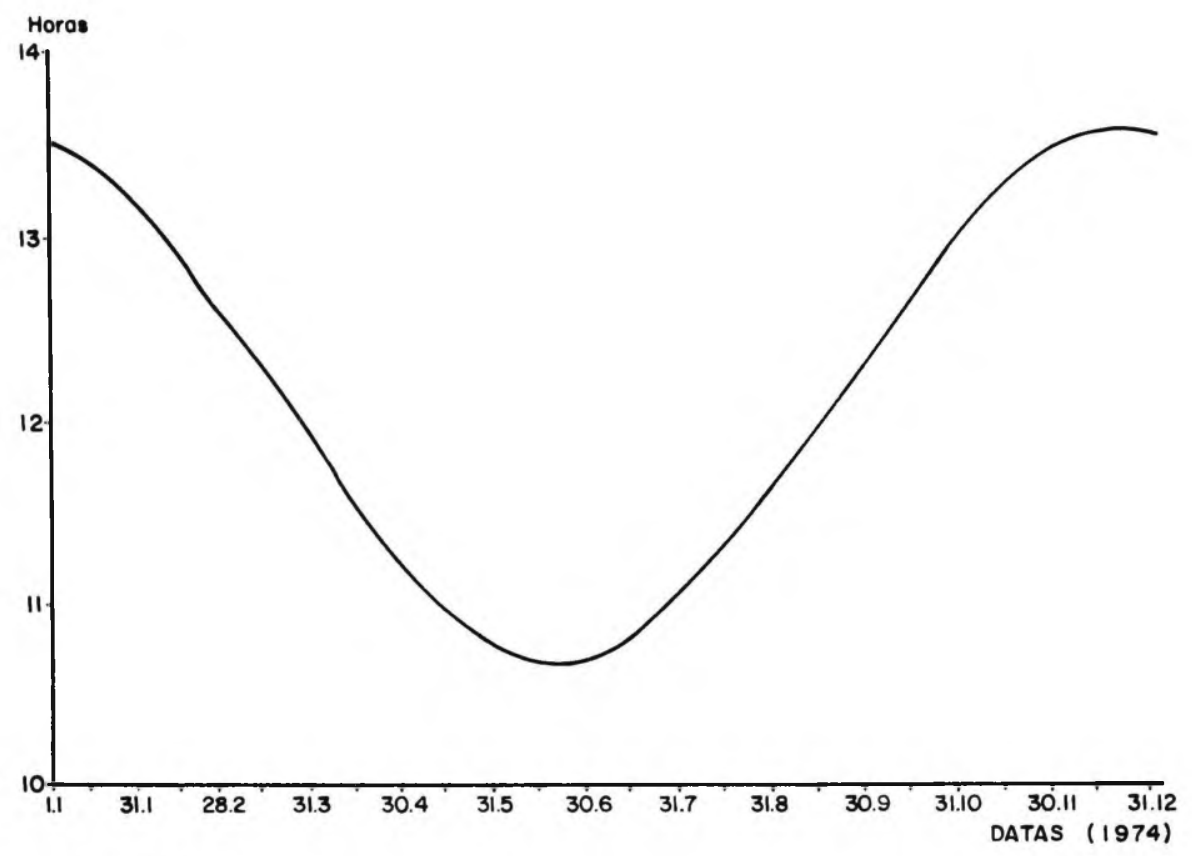

cosol 1 WIIAmorreram

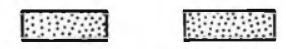

\section{c. $M$ Ull, norrer. - Whe}

cosal $\mathbf{R}$

c. $H \square$

W屋

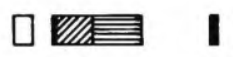

c.늘

casal V

口田翌

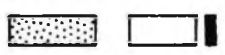

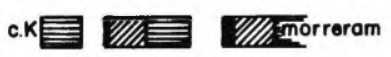

cosal 0

cosal $P$

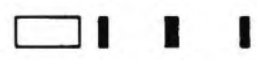

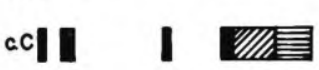

0

Fig. 3 - Comportamento reprodutivo em cativeiro de casais de S. f. brasiliensis, com exceção do casal $C$, que é de $S$. $f$. pelzelni, em função do comprimento dos dias em 1974. 


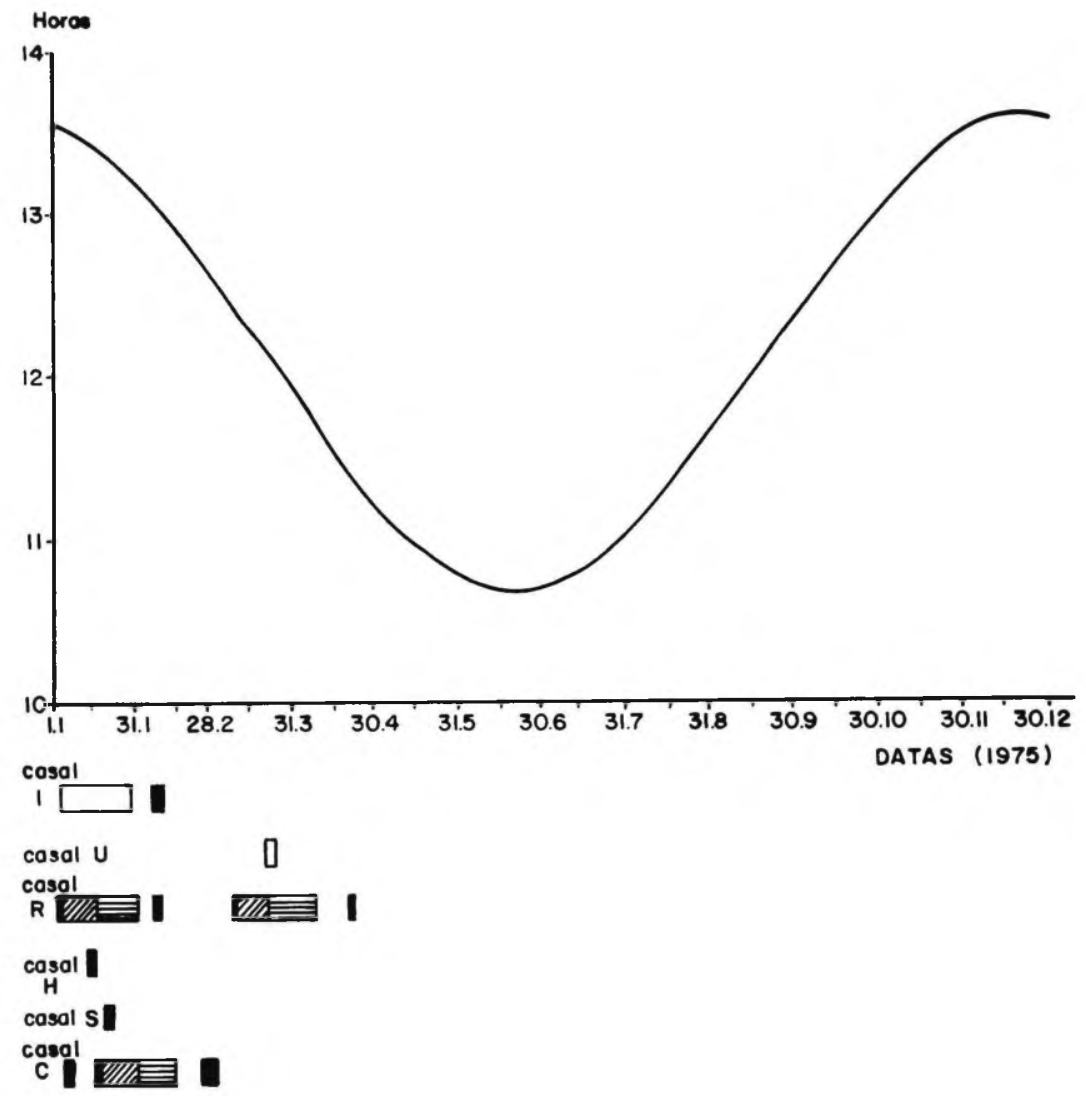

Fig. 4 - Comportamento reprodutivo em cativeiro de casais de $s$. f brasiliensis, com exceção do casal $C$, que é de $S$. $f$ pelzelni, em funçäo do comprimento dos dias em 1975. 


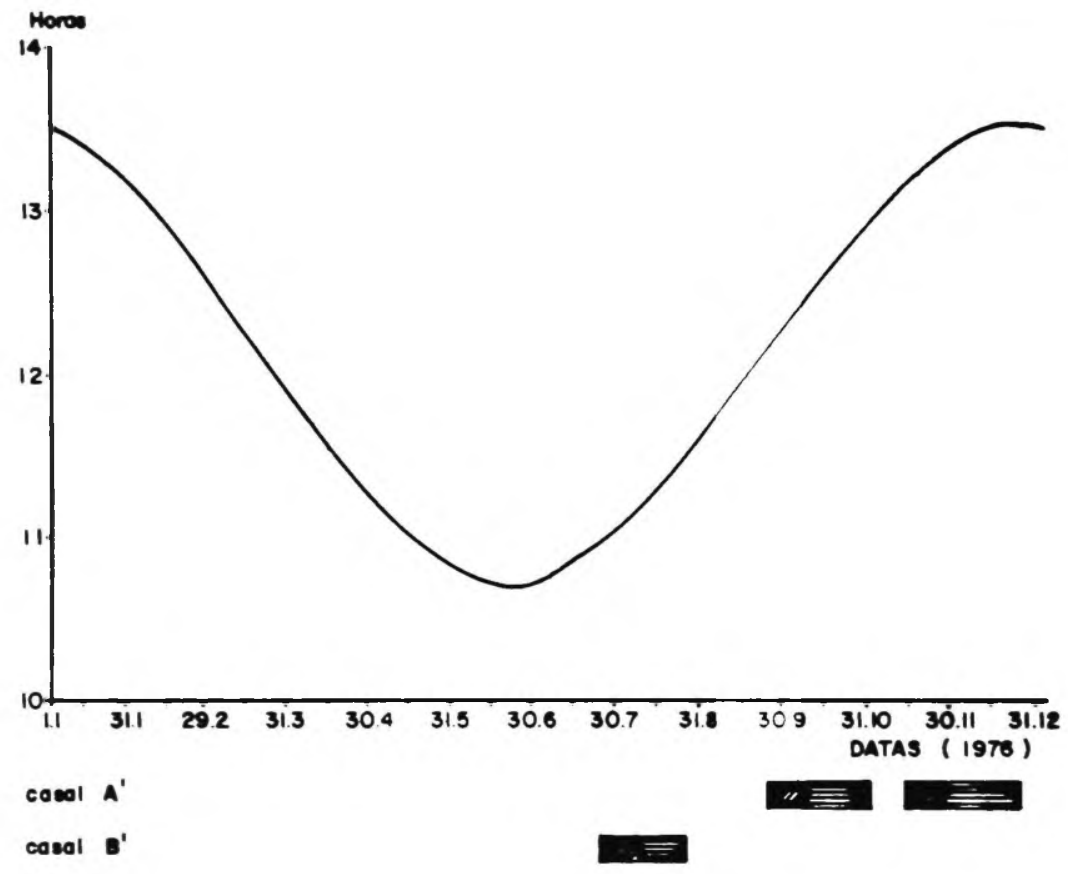

Fig. 5 - Comportamento reprodutivo em função do comprimento dos dias em 1976, de casais de $S$. f. brasiliensis observados em condiçōes naturais. 
Atividade reprodutiva em S. flaveola

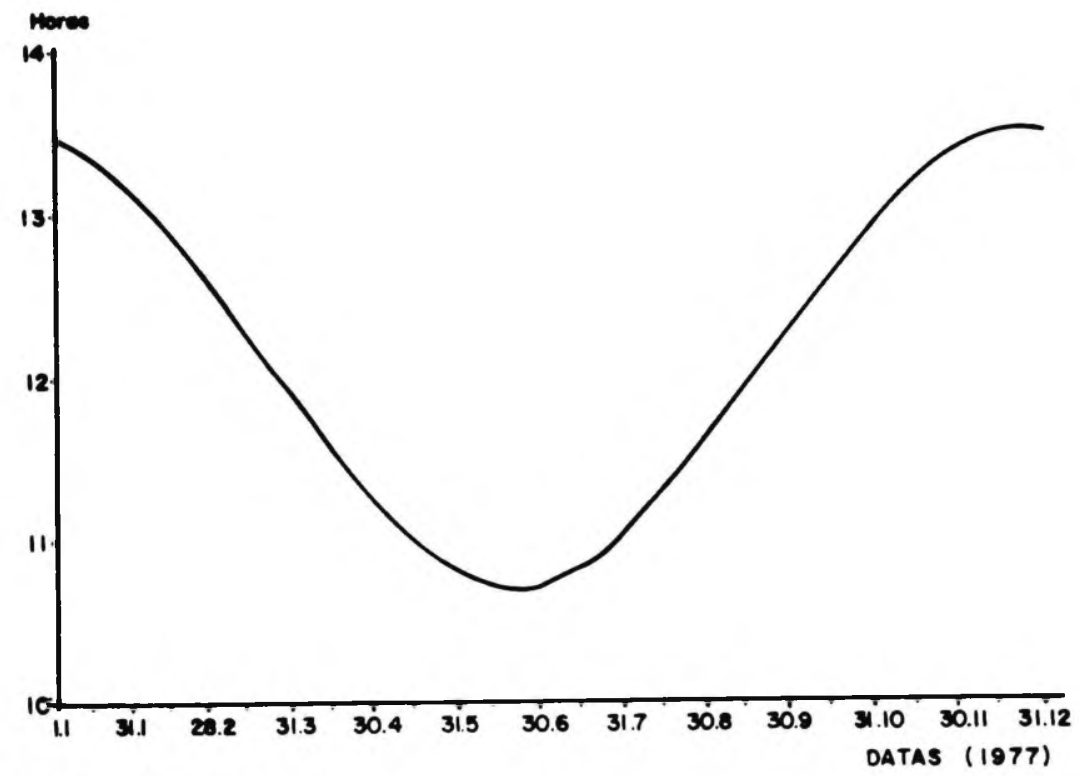

$\cos a c^{\prime}$

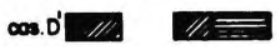

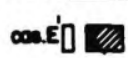

$\infty$ os F'

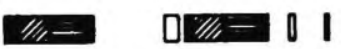

Fig. 6 - Comportamento reprodutivo em função do comprimento dos dias em 1977, de casais de $S$. f. brasiliensis observados em condiçōes naturais. 


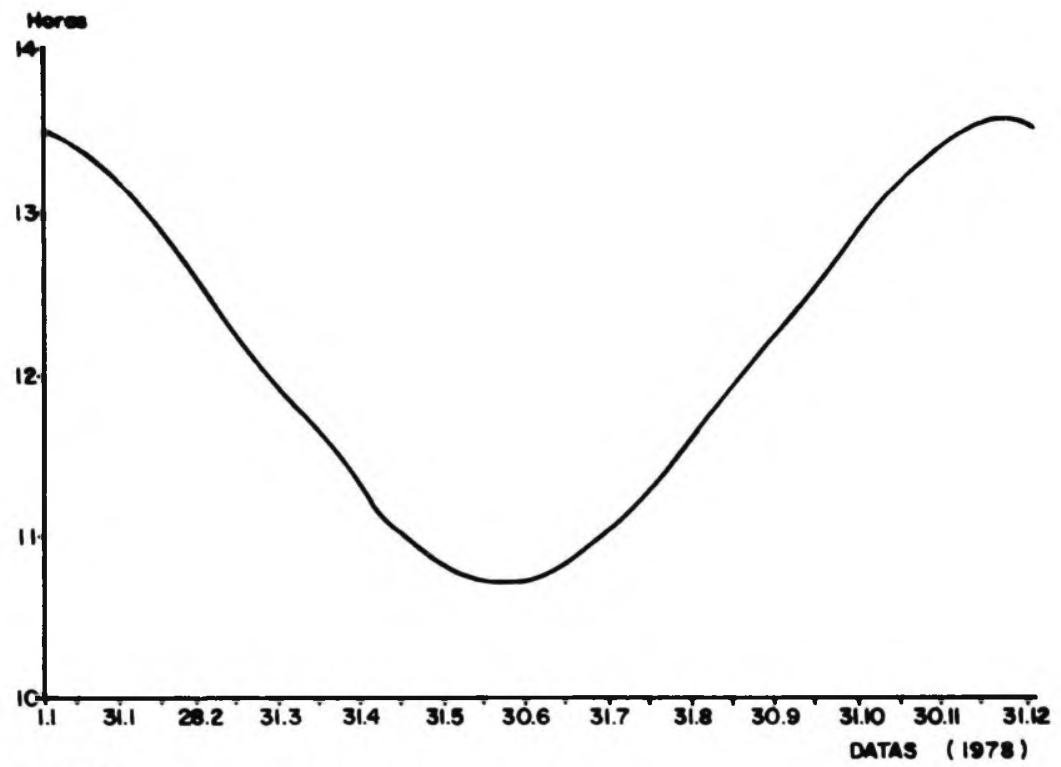

cosal $G^{\prime}$

$\infty \mathrm{N}^{\prime}$

Fig. 7 - Comportamenta reprodutivo em função do comprimento dos dias em 1978 , de casais de $S$. $f$. brasiliensis observados em condições naturais. 
Os períodos de inatividade em alguns casais de $s$. flaveola, após o início da construção do ninho, também ocorrem em outras espécies observadas na natureza, como sporophila nigricollis (Ffrench, 1965) e Spinus tristis (Nickell, 1951) De acordo com Armstrong (1965), tal comportamento se manifes ta quando uma ave não está em estado fisiológico propício pá ra o desencadeamento de toda a sequência de etapas que cons= titui o ciclo reprodutivo.

No caso particular de aves mantidas em cativeiro, há uma aparente propensão para que ocorra uma disrupção nos com plexos padröes de comportamento envolvidos na reprodução, nō tadamente a interrupçao da construção do ninho. Como as se= quências das diferentes fases que formam o comportamento reprodutivo dependem de uma interrelação entre o macho e a fémea, falhas nesta interrelação podem resultar em uma quebra da sequência normal (Lorenz, 1971)

Quanto ao casal de $S$. f. brasiziensis, que procriou em comprimentos de dia relativamente curtos, na natureza, é pro vável que tenha sofrido influência de outros fatores, tais como condiçōes ideais de temperatura e alimentação, o que possibilitou a ocorréncia de ovipostura, seguida de nascimen to e desenvolvimento dos filhotes

AGRADECIMENTOS - $\AA$ José Alberto Marcondes Machado pelas su gestōes, à Eloisa Helena Morgado e Ivan Sazima pela leitura e discussão, à Suzanne Bennett pela correção do abstract, ao Instituto Astronômico e Geofísico da USP pelos dados forneci dos, à Fundação Parque Zoológico de São Paulo e ao Dr. Paulo Nogueira Neto que possibilitaram o presente estudo.

\section{REFERENCIAS}

ARMSTRONG, E.A. 1965. Bird display and behaviour. An intro duction to the study of bird psychology. New York, Dover, $431 \mathrm{p}$.

FFRENCH, R.P. 1965. The nesting behaviour of the Yellow Bellied Seedeater. Caraib.J.Sci., 5:149-156.

IMMELMANN, K. 1971. Ecological aspects of periodic reproduction. In FARNER, D.S.; KING, J.R. \& PARKES, K.C. eds Avian Biology. New York, Academic Press, v.1, p. 341-389

LACK, D. 1968. Ecological adaptations for breeding in birds. London, Methuen, $409 \mathrm{p}$.

LORENZ, K. 1971. Studies in animal and human behaviour. Cambridge, Harvard University Press. v.l, 403 p.

NICKELL, W.P. 1951. Studies of habitats, territory, and nests of the Eastern Goldfinch. Auk, 68:447-470.

SKUTCH, A.F 1950. The nesting seasons of Central American birds in relation to climate and supply, Ibis, 92:185-222

WELTY, J.C. 1962. The life of birds. Philadelphia, Saunders, 546 p. 